\title{
Efficacy of Temporalis Myofascial Flap in Maxillofacial Reconstruction
}

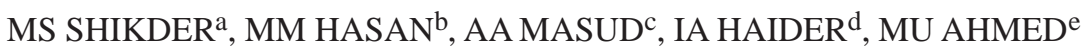

\begin{abstract}
Summary:
Temporalis myofascial flap holds great promise for the reconstruction of various defects of the maxillofacial region for its dependable blood supply, proximity to the maxillofacial region, possibility to mobilize it to the oral cavity and its fanned out nature.
\end{abstract}

The purpose of the study was to evaluate the efficacy of temporalis myofascial flap in maxillofacial reconstruction.This cross sectional study was carried out in the Department of Oral and Maxillofacial Surgery, Dhaka Dental College and Hospital from January 2011 to December 2011. Nineteen patients (10 male and 9 female), age ranging from 19 to 55 years with medium to large defect $(>8 \mathrm{~cm})$ were selected for this study. After surgical resection

\section{Introduction:}

Reconstruction should strive to restore the maxillofacial form, versatility of tissues, oral competence and oral cavity function and thus allowing the patients to meet the social needs. ${ }^{1}$ Various defects in orofacial region due to tumor extirpation, trauma or congenital defect requires subsequent reconstruction. The choice of reconstructive technique depends upon numerous factors related to each individual case. These factors include the site, size and composition of the defect. A large variety of techniques are available for reconstruction of the defects. Each technique has its advantages and limitations. The simplest surgical procedure with good surgical, functional and esthetic

a. Dr. Muhammad Sohag Shikder, Maxillofacial Surgeon.

b. Dr. Md. Mokerrom Hasan, Lecturer, Dept. of Oral and Maxillofacial Surgery, DDCH.

c. Dr. Abdullah Al Masud, Assistant Professor, Dept. of Oral and Maxillofacial Surgery, DDCH.

d. Dr. Ismat Ara Haider, Associate Professor, Dept. of Oral and Maxillofacial Surgery, DDCH.

e. Professor Dr. Mohiuddin Ahmed, Professor and Head, Dept. of Oral and Maxillofacial Surgery, DDCH.

Address of the correspondent: Dr. Md. Sohag Shikder, HouseDCC-95, $4^{\text {th }}$ floor, Sharif Miar Bari, Poolpar, Ibrahimpur, Kachukhet Cantonment, Dhaka, E-mail shikderd36@yahoo.com Mob: 01716604751

Received: 11April, 2013

Accepted: 8 February, 2014 of the pathological lesion, the TMF was exposed by a hemicoronal incision with a preauricular extension. The muscle was rotated to oral cavity and sutured with defect margin. Post-operatively the patient were clinically evaluated at 1, 2, 3 weeks and 1 and 3 months. Complete flap take was observed in $84 \%$ cases where as partial flap take was seen in $16 \%$ cases. Mouth opening restriction were 2-3 $\mathrm{mm}$ in 10 cases and $5-8 \mathrm{~mm}$ in 5 cases.Temporalis myofascial flap is a useful, reliable and versatile option for reconstruction of moderate to large sized defects. This flap provide abundant tissue, with minimum to no functional morbidity or esthetic deformity in donor site.

Key words: TMF-temporalis myofascial flap.

(J Banagladesh Coll Phys Surg 2014; 32: 78-83)

results and having minimum morbidity should be adopted. Several soft tissue flaps can be used for the reconstruction of defect in the orofacial region. These include local, regional and free flaps. Local flap is enough to reconstruct the small defect but for moderate to large defect regional or free flaps are often necessary. Of the various regional flaps temporalis myofascial flap provides one of the best option because of its reliable vascularity, adequate bulk and proximity to the defects in orofacial region. ${ }^{2}$ Since the viability of the flap is excellent, post operative radiotherapy can be taken up much earlier than any types of reconstruction. ${ }^{3}$

The apparent advantages of temporalis myofascial flap (TMF) is evident from other studies in different countries as it is easy in performing the technique, proximity of the flap to the oral cavity, minimal or no donor side morbidity and minimal post operative complication. But there is no systematic study to verify its potential and outcome in Bangladesh is available for reconstruction of the defects. In a study John $\mathrm{F}$ arvier showed that for the repair of the adult cleft palate the transantral transfer of TMF is a single stage procedure with low morbidity and few complication. ${ }^{4}$ The present study was designed to find out the efficacy of temporalis myofascial flap for reconstruction of various defects of maxillofacial region after tumour surgery in the department of Oral and Maxillofacial Surgery (OMS), Dhaka Dental College \& Hospital (DDCH). 
The aim of this study is to evaluate the effectiveness of temporalis myofascial flap in reconstruction purpose depending on size of the defect, length of the flap and operating time.

\section{Material and Methods:}

This Prospective type of cross-sectional study was carried out in the department of oral and maxillofacial surgery, Dhaka Dental College and Hospital from January 2011 to December 2011. Patient admitted in the Department of Oral and Maxillofacial Surgery, Dhaka Dental College and Hospital with orofacial tumor and deformity requiring ablative surgery were selected for this study. Surgical planning was done and the defect was categorized as medium to large defect (medium defect $4-8 \mathrm{~cm}$, large defect $>8 \mathrm{~cm}$ ). Total of 19 patients were selected including tumor and cleft palate defect.

A detailed medical history of the selected patient and thorough clinical examination was done. In all cases site and size of the lesion and probable defect was measured preoperatively. After surgical resection of the pathological lesion the defect to be reconstructed was measured. The TMF was exposed using a hemicoronal incision with a pre-auricular extension. Dissection was carried out at a subgaleal level. It was then mobilized subperiosteally from its deep origin in the temporal fossa. Anteriorly the muscle was elevated from lateral aspect of the orbit and inferiorly down to the temporal crest. The temporal fascia was then incised transversely $2 \mathrm{~cm}$ above the zygomatic arch so as not to injure the zygomatic branch of facial nerve. The zygomatic arch was exposed and divided as far forward and as far posteriorly as possible to allow the muscle to be rotated in to the oral cavity. The muscle was sutured to the defect margin of the oral cavity with the temporal fascia facing the oral cavity. The donor site was closed in layers over a suction drain. Post-operatively all the patients were clinically evaluated after 1st, 2nd, 3rd week, 1st and 3rd month. Post-operatively patient was evaluated on the basis of flap success (flap color, epithelialization, and adaptation), flap failure (Infection, wound dehiscence, sloughing and fistula), complication (mouth opening/trismus, facial deformity) and donor site morbidity (Infection, deformity). Per-operative evaluation was done with defect size, flap size and operating time.

In post-operative evaluation flap viability was assessed by pink color, pale color and blue with pink color. Epithelialization was assessed by homogenosity of the flap with adjacent mucosa.
In complication, mouth opening was measured by inter incisal opening with scale and facial deformity was judged comparing with opposite side of the face with visual examination.

Donor site morbidity was expressed by infection which indicated any sort of secretion from donor site and deformity was assessed with opposite temporal fossa. The data was collected in preformed questionnaire. The data was analyzed with statistical software (spss va 20) and was represented with table, diagram.

\section{Result:}

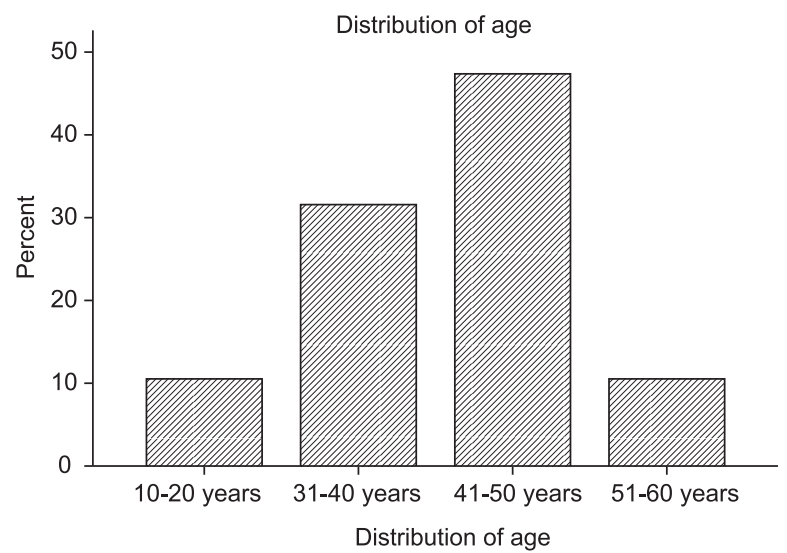

Fig-.1: A bar diagram showing distribution of age. The age range of the study population was 10-60 years but the lowest age was 19 and the highest was 55 years. The highest percentage (47.4\%) of the subjects was from the age group 41 to 50. There was no subject available for the age group 21-30 years.

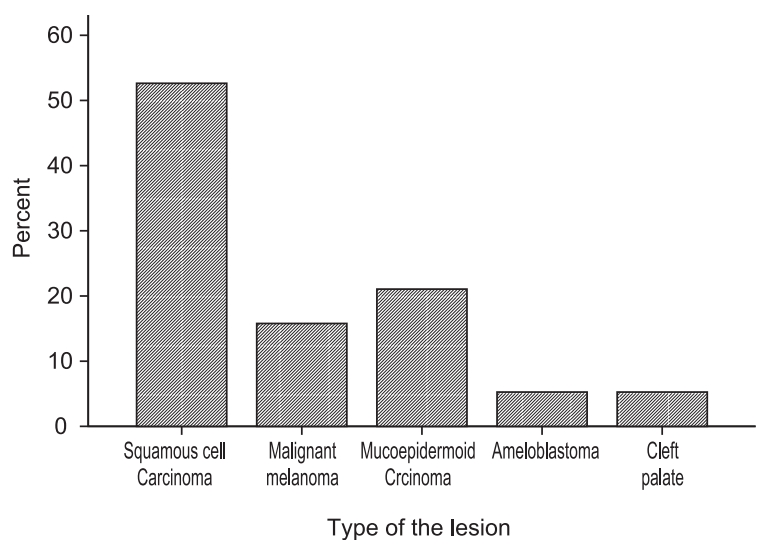

Fig-.2: A bar diagram showing the type of lesion. Sq. cell carcinoma is more frequent (52.6\%) among all other lesions. Then comes mucoepidermoid carcinoma and malignant melanoma accordingly. Ameloblastoma and cleft palate accounts the least percentage. 


\section{Table-I}

Post operative outcome of the subjects after 3 weeks and 3 months post operatively.

\begin{tabular}{|c|c|c|c|}
\hline \multicolumn{2}{|c|}{ Postoperative outcome } & \multicolumn{2}{|c|}{ Postoperative duration } \\
\hline & & $\begin{array}{l}\text { After } 3 \\
\text { weeks }\end{array}$ & $\begin{array}{l}\text { After } 3 \\
\text { months }\end{array}$ \\
\hline \multirow[t]{2}{*}{ Flap color } & Pink & $18(94.7 \%)$ & $19(100 \%)$ \\
\hline & Pale-pink & $1(5.3 \%)$ & $00(0 \%)$ \\
\hline Blue with pink & $0(0 \%)$ & $00(0 \%)$ & \\
\hline \multirow[t]{2}{*}{ Epithelialization } & Yes & $8(42.1)$ & $19(100 \%)$ \\
\hline & No & 11(57.9) & $00(0 \%)$ \\
\hline \multirow[t]{2}{*}{ Adaptation } & Yes & $17(89.5 \%)$ & $19(100 \%)$ \\
\hline & No & $02(10.5 \%)$ & $00(0 \%)$ \\
\hline \multirow[t]{2}{*}{ Post-op. infection } & Yes & $00(0 \%)$ & $00(0 \%)$ \\
\hline & No & $19(100 \%)$ & $19(100 \%)$ \\
\hline \multirow[t]{2}{*}{ Sloughing } & Yes & $11(57.9 \%)$ & $00(0 \%)$ \\
\hline & No & $08(42.1 \%)$ & $19(100 \%)$ \\
\hline \multirow[t]{2}{*}{ Wound dehiscence } & Yes & $00(0 \%)$ & $00(0 \%)$ \\
\hline & No & $19(100 \%)$ & $19(100 \%)$ \\
\hline \multirow[t]{2}{*}{ Fistula } & Yes & $01(5.3 \%)$ & $00(0 \%)$ \\
\hline & No & $18(94.7 \%)$ & $19(100 \%)$ \\
\hline \multirow[t]{2}{*}{ Facial deformity } & Yes & $03(15.8 \%)$ & $00(0 \%)$ \\
\hline & No & $16(84.2 \%)$ & $19(100 \%)$ \\
\hline \multirow[t]{2}{*}{ Donor site infection } & Yes & $00(0 \%)$ & $00(0 \%)$ \\
\hline & No & $19(100 \%)$ & $19(100 \%)$ \\
\hline \multirow[t]{2}{*}{ Donor site deformity } & Yes & 19(100\%) & $19(100 \%)$ \\
\hline & No & $00(0 \%)$ & $00(0 \%)$ \\
\hline
\end{tabular}

Table-I: shows post operative outcome with different parameters after 3 weeks and after 3 months. All subjects show pink color flap, proper adaptation without any fistula but show donor site deformity in all cases after 3 months.

\section{Table-II}

\begin{tabular}{lcccc} 
& \multicolumn{4}{c}{ One-Sample statistics } \\
& $\mathrm{N}$ & Mean & $\begin{array}{c}\text { Std. } \\
\text { Deviation }\end{array}$ & $\begin{array}{c}\text { Std. Error } \\
\text { Mean }\end{array}$ \\
\hline $\begin{array}{l}\text { Preoperative mouth } \\
\text { opening }\end{array}$ & 19 & 32.1053 & 7.68038 & 1.76200 \\
$\begin{array}{l}\text { Postoperative } \\
\text { mouth opening } \\
\text { (after 3 months) }\end{array}$ & 19 & 30.0000 & 4.83046 & 1.10818 \\
\hline
\end{tabular}

Table-II Shows mean pre \& post operative mouth opening with standard deviation. It showed that mean preoperative mouth opening was $32.10 \mathrm{~mm}$ and $\mathrm{SD} \pm 7.68$ where as mean post operative mouth opening was $30.00 \mathrm{~mm}$ and $\mathrm{SD} \pm 4.83$. that is mean difference was $2.1 \mathrm{~mm}$.

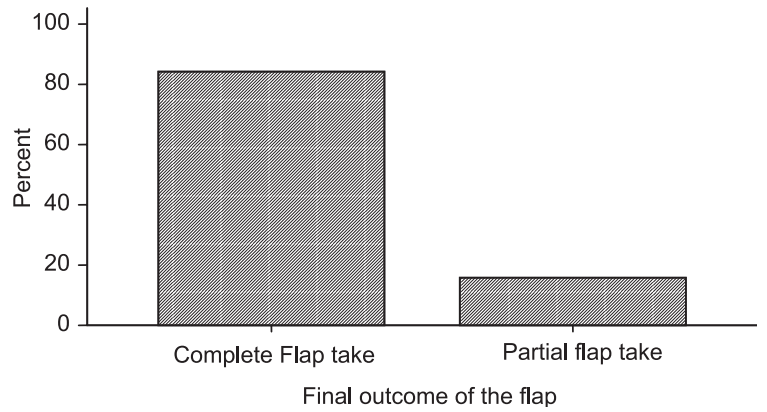

Fig.-3: A bar diagram showing final outcome of the flap where complete take is observed in $84 \%$ cases and partial take was observed in 16\% cases.

\section{Illustration}

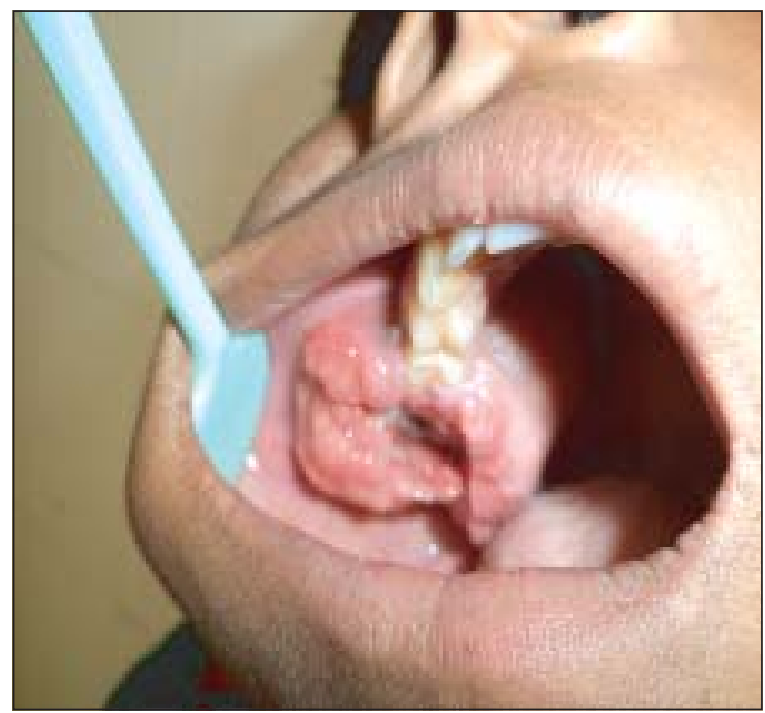

Pre operative: A case of Right maxillary ameloblastoma involving buccal and palatal aspect of right maxilla from right upper fist premolar to retromolar region.

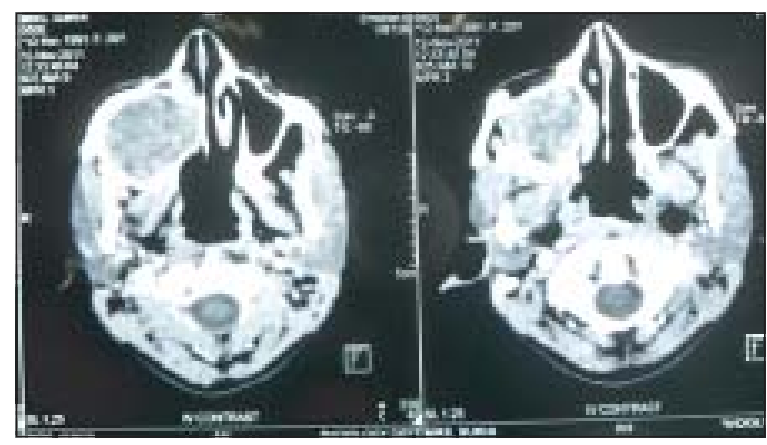

Pre operative: Axial section of Radiograph showed involvement of right maxilla with mixed density of right maxillary sinus but no erosion of buccal or posterior wall and lateral wall of the nose is seen 


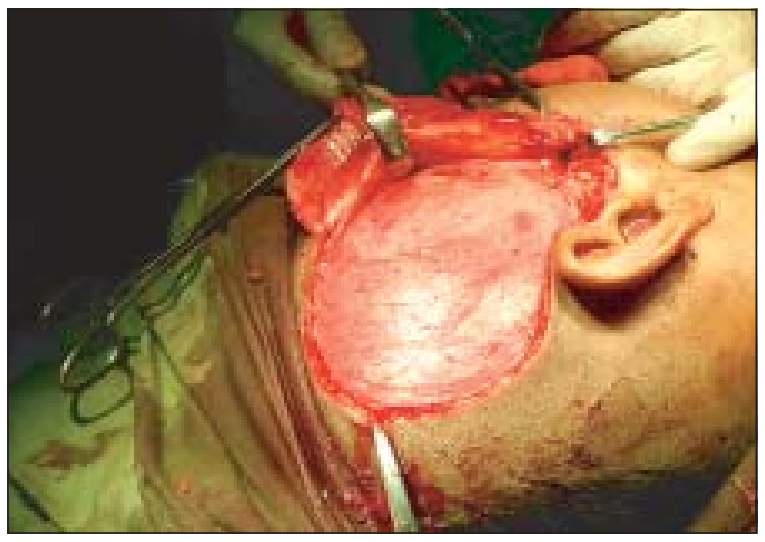

Per operative: Incision and exposure of TMF with hemicoronal incision with preauricular extension

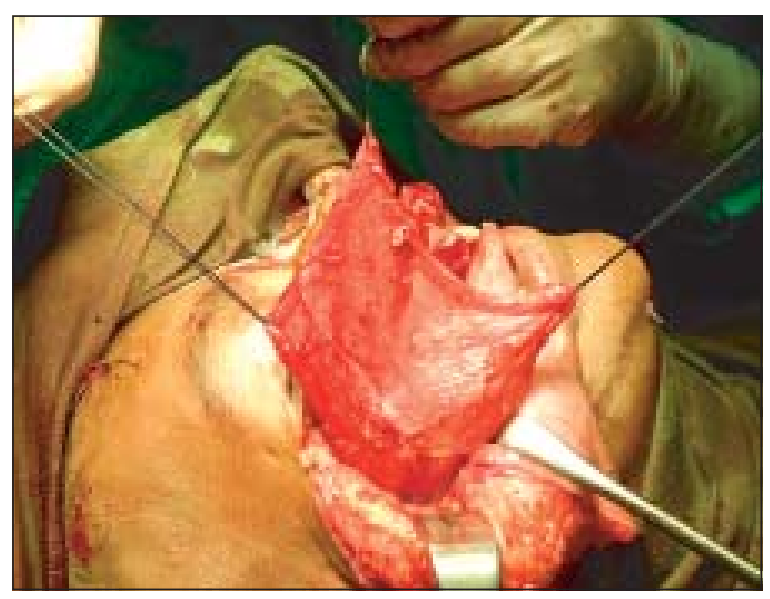

Per operative: Transfer of TMF with in the oral cavity through the resection of zygomatic arch.

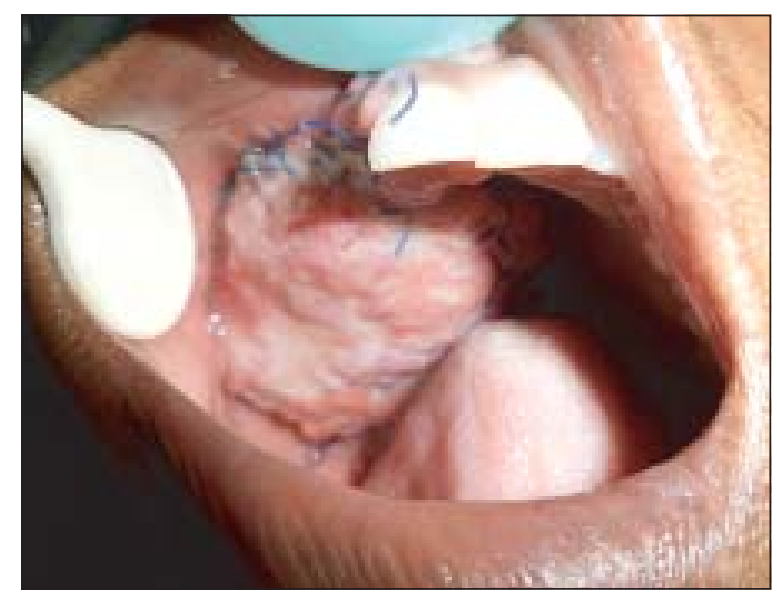

Post operative: After three weeks proliferative stage of TMF with sloughing of superficial fascia

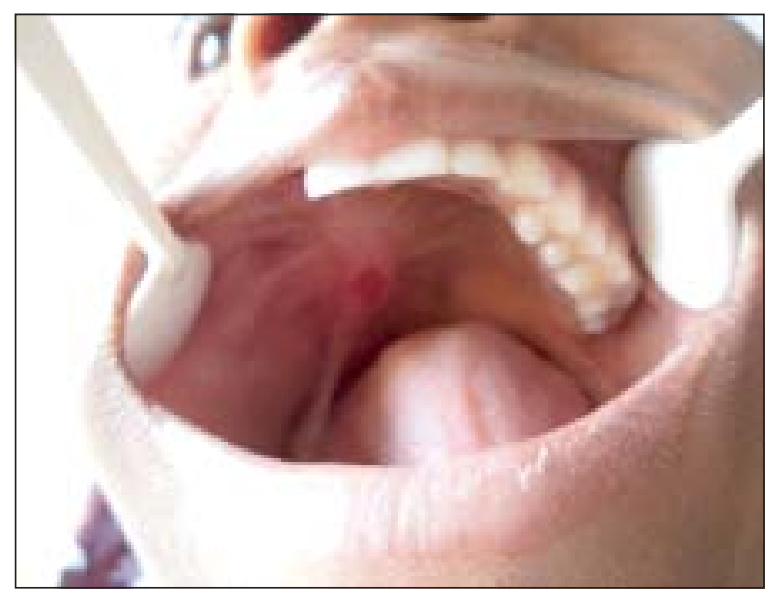

Post operative: After three months complete healing of TMF with epithelialisation

\section{Discussion:}

Surgical treatment of malignancies in the maxillofacial region has improved with advances in reconstructive techniques with a thorough knowledge of the anatomy of the flaps and their clinical application. Many different reconstructive procedures are available for this purpose. Local flaps can be used successfully for small defects. However, for moderate to large defects free or regional flaps are often necessary to reconstruct the defects. ${ }^{2}$ Microvascular free flap might be the best choice for reconstruction but difficult to perform due to limited facilities in the place of study ( large patient que, logistics support ) and expertise. Where as distant flaps are not always a good option for maxillofacial defects particularly for palate, check due to their long distance, excessive bulk and donor site morbidity.

The TMF is simple with short harvesting time, versatile, regional proximity, mobility of a thin tissue mass on a vascular pedicle and lacks functional morbidity at donor site with acceptable cosmesis which are significant advantages of the use of this flap. ${ }^{5}$ This study used TMF for maxillofacial defect in 19 patients, age ranges from 19 to 55 years. Among them 41-50 years age groups(Fig.1) comprises the highest percentage (47.4\%). Among the study subject $52.63 \%$ were male and $47.37 \%$ were female and male female ratio was 10:9. Estelles Freriol et al. (2005) used TMF in 22 patients where male were 14 and female were 8 with a age range between 37 to 73 years. ${ }^{6}$

Among 19 cases most of the cases (52.6\%) were squamous cell carcinoma then comes mucoepidermoid 
carcinoma ,malignant melanoma and ameloblastoma (Fig.2). Most involved site was palate particularly midpalate. In 38 cases of Hoyo et al. for TMF reconstruction, 23 cases were sq. cell carcinoma and most involving site was maxillary sinus. ${ }^{7}$ The study result of Hoyo et al. showed that after surgical resection of maxillary carcinoma TMF might be the treatment of choice for reconstruction which supports the presented study.

TMF flap can be used for the reconstruction of mandibular soft tissue upto the canine region. ${ }^{8}$ In the present study most of the site of the defect was in the midpalate and cheek.

Operating time is an important factor for both the patient and for the surgery out come. In a tertiary hospital like $\mathrm{DDCH}$ where patient flow is higher, operating time needed to be averaged to meet the high patient flow. In all of the study cases mean operating time was 126.31 minutes with a SD of 12.56 minutes. Distant flap and microvascular flap procedure would have been more elaborate and time consuming.

Viability of the flap was observed by color of the flap. A flap that had intact blood supply with good perfusion showed normal color that is pink while flap with poor perfusion showed pale color and flap with venous congestion showed bluish color. ${ }^{1}$ In the present study after from $1^{\text {st }}$ POD to 3 weeks $94.7 \%$ showed pink color which became $100 \%$ after 3 months. Ahmed et al. got $90 \%$ success rate of flap while $10 \%$ of failure due to diabetes. ${ }^{1}$ Abu baker in 2002 also get $100 \%$ success rate with his study. ${ }^{2}$

Flap adaptation at the defect margin is an important criteria for flap success. In 3 weeks follow up 17 (89.5\%) showed good adaptation at the flap margin but at 3 months follow up all the cases $19(100 \%)$ showed good adaptation. Regarding epithelialisation of the flap $42.1 \%$ of the cases showed surface epithelialisation within 3 weeks and $100 \%$ achieved surface epithelialisation in 3 months follow up. The epithelialization of the flap took place in around 3 weeks time in the study of Mani and Panda. ${ }^{3}$ This result differs with the study of Mani and may be due to the patients condition. Sloughing of temporal fascia frequently occurred in the Hoyo study. ${ }^{7}$ In our study, fascia sloughing occurred in all (100\%) cases up to 3 weeks. Wound dehiscence is major complication of flap outcome. Dehiscence may result in flap failure. But in this study during follow up period wound dehiscence was nil. No dehiscence or fistula occurred in Hoyo study. ${ }^{7}$ Hoyo study matches with the present.

Mouth opening frequently reduces after maxillectomy if done without reconstruction. Ahmed et al. showed post operative mouth opening restriction only in 2 out of 28 cases, but Naaj et al. got mouth opening limitation in most cases in their study. ${ }^{1,9}$ Pre and postoperative mouth opening was studied and compared in the present study. It showed that mean preoperative mouth opening was $32.10 \mathrm{~mm}$ with SD of 7.68, where as mean post operative mouth opening was $30.00 \mathrm{~mm}$ with SD of 4.83 . So, mean difference was $2.1 \mathrm{~mm}$ which was probably due to inadequate post operative jaw exercise.

Before harvesting any flap donor site morbidity must be kept in mind. All patients had donor site deformity which was also present in the study of Naaj et al. due to lack of tissue bulk that had been harvested. ${ }^{9}$ Depression of the donor site was found in all of the $(100 \%)$ cases upto 3 months follow-up period. But the deformity was masked in all cases by the hair line. This is an advantage of TMF comparing to others.

Flap success depends on all the factors described above. Temporalis muscle flap success rate was $80 \%$ with two minor complication that resolved without damage to the flap in a study. ${ }^{9}$ Only one patient out of 26 had complete flap necrosis in Colmenero study. ${ }^{10}$ In Hoyo study there was no partial or total flap necrosis in 38 cases $^{7}$. In the present study complete flap take occurred in $84 \%$ of the cases after complete follow up and only $6 \%$ showed partial flap take and it was due to the marginal necrosis of the flap.

\section{Conclusion:}

Temporalis myofascial flap is an axial pattern flap, which is an excellent choice for oral and maxillofacial reconstruction, especially defects of palate and cheek. Temporalis myofascial flap is far less bulky, more pliable, non-hair bearing and in close proximity to the oral cavity. Use of this muscle flap does not impair mandibular function. The depression is minimal and the hair covers most of the donor area.

\section{References:}

1. Ahmad S, Warraich RA, Abid H. The versatility of Temporalis muscle flap in reconstruction of maxillofacial region. Annals 2009; 15: 1. 
2. Abubaker A O, Mustafa B, Abouziga. The temporalis muscle flap in reconstruction of intra oral defects: An appraisal of technique. Oral surg oral Med oral Pathol oral Radiol Endod 2002; 94: 24-30.

3. Mani V, Panda A K. Versatility of temporalis myofascial flap in maxillofacial reconstruction-analysis of 30 cases. Int J Oral Maxillofac Surg 2003; 32: 368-372.

4. John F. Arvier, Motiur R. Molla, S. M. Iqbal Shaheed, Kerry Lanza Barry Fitzpatrick Trans-antral temporalis transfer for the repair of adult cleft palates. Australian Dental Journal 1997;42:(5):307-14

5. Demas P N, Sotereanos G C. Transmaxillary temporalis transfer for reconstruction of a large palatal defect. J Oral Maxillofac Surg 1989; 47: 197-202.
6. Eestelles Ferriol J E, Liatas M C, Ferrer Ramirez M J. Temporalis myofascial flap: technique description and results in our patient. Acta otorrinolarissgol Esp 2005; 56: 257-260.

7. Dell hoyo J A, Sanroman J F, Gil-diez J L. The temporalis muscle flap. J Oral Maxillofac Surg 1994; 52: 143-147.

8. Bradley P, Brockbank J. The temporalis muscle flap in oral reconstruction. J Max-Fac Surg1981; 9: 139-145.

9. Naaj I A, Leiser Y, Liberman R, Peled M. The use of temporalis myofascial flap in oral cancer patient. J.oral maxillofac surg; 68: 578-583.

10. Colmenero C, MartorellA V, Colmenero B, SierraI I. Temporalis myofascial flap for maxillofacial reconstruction. J Oral Maxillofac Surg 1991; 49: 1067-1073. 